\title{
Dirichlet problems for stationary von Neumann-Landau wave equations
}

\section{Zeqian Chen}

Wuhan Institute of Physics and Mathematics, Chinese Academy of Sciences, 30 West District, Xiao-Hong-Shan, P.O.Box 71010, Wuhan, China

E-mail: zqchen@wipm.ac.cn

\begin{abstract}
It is known that von Neumann-Landau wave equation can present a mathematical formalism of motion of quantum mechanics, that is an extension of Schrödinger's wave equation. In this paper, we concern with the Dirichlet problem of the stationary von Neumann-Landau wave equation:$$
\left\{\begin{array}{l}
\left(-\triangle_{x}+\triangle_{y}\right) \Phi(x, y)=0, \quad x, y \in \Omega, \\
\left.\Phi\right|_{\partial \Omega \times \partial \Omega}=f,
\end{array}\right.
$$

where $\Omega$ is a bounded domain in $\mathbf{R}^{n}$. By introducing anti-inner product spaces, we show the existence and uniqueness of the generalized solution for the above Dirichlet problem by functional-analytic methods.
\end{abstract}

AMS classification scheme numbers: 35Q40, 35D05 


\section{Introduction}

In the most general form, Heisenberg's equation and Schrödinger's equation can be written as follows

$$
i \hbar \frac{\partial \hat{O}(t)}{\partial t}=[\hat{O}(t), \hat{H}]
$$

and

$$
i \hbar \frac{\partial|\psi(t)\rangle}{\partial t}=\hat{H}|\psi(t)\rangle
$$

respectively, where $\hat{H}$ is the Hamiltonian of the system. As is well known, these two forms for the equations of motion of quantum mechanics are equivalent [3]. Of these, the Schrödinger form seems to be the more useful one for practical problems, as it provides differential equations for wave functions, while Heisenberg's equation involves as unknowns the operators forming the representative of the dynamical variable, which are far more numerous and therefore more difficult to evaluate than the Schrödinger unknowns [6]. Also, determining energy levels of various dynamic systems is an important task in quantum mechanics, for this solving Schrödinger's wave equation is a usual way. Recently, Fan and Li [4] showed that Heisenberg's equation can also be used to deduce the energy level of some systems. By introducing the conception of invariant 'eigen-operator', they derive energy-level gap formulas for some dynamic Hamiltonians. However, their 'invariant eigen-operator' equation involves operators as unknowns, as similar to Heisenberg's equation, and hence is also difficult to evaluate in general.

Recently, it is shown by the author [2] that von Neumann-Landau equation for wave functions is an extension of Schrödinger's wave equation and can be used to determine energy-level gaps of the system. Contrary to Schrödinger's wave equation, vNLW is on 'bipartite' wave functions. It is shown that these 'bipartite' wave functions satisfy all the basic properties of Schrödinger's wave functions which correspond to those 'bipartite' wave functions of product forms. Precisely, consider the quantum system of a single particle. Note that the Hamiltonian for a single particle in an external field is

$$
\hat{H}(\vec{x})=-\frac{\hbar^{2}}{2 m} \nabla_{\vec{x}}^{2}+U(\vec{x})
$$

where $\nabla_{\vec{x}}^{2}=\partial^{2} / \partial x_{1}^{2}+\partial^{2} / \partial x_{2}^{2}+\partial^{2} / \partial x_{3}^{2}, U(\vec{x})$ is the potential energy of the particle in the external field, and $\vec{x}=\left(x_{1}, x_{2}, x_{3}\right) \in \mathbf{R}^{3}$. The Schrödinger's wave equation describing dynamics of the particle is

$$
i \hbar \frac{\partial \psi(\vec{x}, t)}{\partial t}=\hat{H}(\vec{x}) \psi(\vec{x}, t)=-\frac{\hbar^{2}}{2 m} \nabla_{\vec{x}}^{2} \psi(\vec{x}, t)+U(\vec{x}) \psi(\vec{x}, t) .
$$

The state of the particle can be described by a definite wave function $\psi$ of Equation (1.4), whose stationary states determine its energy levels. Moreover, the expectation value of an observable $\hat{Q}$ in the state corresponding to $\psi$ is determined by $\langle\hat{Q}\rangle_{\psi}=\langle\psi|\hat{Q}| \psi\rangle$. 
On the other hand, von Neumann-Landau equations for wave functions are the following wave equations - von Neumann-Landau wave equations (vNLW):

$$
i \hbar \frac{\partial \Psi(\vec{x}, \vec{y} ; t)}{\partial t}=(\hat{H}(\vec{x})-\hat{H}(\vec{y})) \Psi(\vec{x}, \vec{y} ; t),
$$

where $\Psi(\vec{x}, \vec{y} ; t) \in L_{\vec{x}, \vec{y}}^{2}$. Contrary to Schrödinger's wave equation Eq.(1.4) for 'onepartite' wave functions $\psi(\vec{x}) \in L_{\vec{x}}^{2}$, the wave equation Eq.(5) is an differential equation for 'bipartite' wave functions $\Psi(\vec{x}, \vec{y})$, which, replacing $\hat{H}(\vec{x})+\hat{H}(\vec{y})$ by $\hat{H}(\vec{x})-\hat{H}(\vec{y})$, is also different from Schrödinger's wave equation for two particles.

We would like to mention that Eq.(1.5) has been presented by von Neumann [7] and Landau [6] giving the change in the density matrix with time. In [2], the author regard Eq.(1.5) as a wave equation but not a equation for density functions. This is the key point which is distinct from [7] and [6]. Then, the author shows that the von NeumannLandau wave equation Eq.(1.5) is mathematically an extension of Schrödinger's wave equation Eq.(1.4) and a suitable form for motion of quantum mechanics as a 'bipartite' wave equation which presents a mathematical expression of wave-particle duality.

Indeed, since

$$
\frac{\partial|\Psi(\vec{x}, \vec{y} ; t)|^{2}}{\partial t}=2 \operatorname{Re}\left[\Psi^{*}(\vec{x}, \vec{y} ; t) \frac{\partial \Psi(\vec{x}, \vec{y} ; t)}{\partial t}\right]
$$

it is concluded from Eq.(1.5) that

$$
\frac{\partial}{\partial t} \int|\Psi(\vec{x}, \vec{y} ; t)|^{2} d^{3} \vec{x} d^{3} \vec{y}=0 .
$$

This means that, if this wave function $\Psi$ is given at some instant, its behavior at all subsequent instants is determined. Hence, Schrödinger's wave equation is a special case of the wave equation Eq.(1.5) with initial values of product form $\Psi(\vec{x}, \vec{y} ; 0)=\psi(\vec{x}) \psi^{*}(\vec{y})$, because in this case $\Psi(\vec{x}, \vec{y} ; t)=\psi(\vec{x}, t) \psi^{*}(\vec{y}, t)$ with $\psi(\vec{x}, t)$ satisfying Eq.(1.4) and $\psi(\vec{x}, 0)=\psi(\vec{x})$ and vice versa.

In this paper, we concern with the stationary von Neumann-Landau wave equation. Similar to Laplace equation, we study the Dirichlet problem for the stationary von Neumann-Landau wave equation:

$$
\left\{\begin{array}{l}
\left(-\triangle_{x}+\triangle_{y}\right) \Phi(x, y)=0, \quad x, y \in \Omega, \\
\left.\Phi\right|_{\partial \Omega \times \partial \Omega}=F
\end{array}\right.
$$

where $\Omega$ is a bounded domain in $\mathbf{R}^{n}$ and $\triangle_{x}=\sum_{j=1}^{n} \frac{\partial^{2}}{\partial x_{j}^{2}}$ for $x=\left(x_{1}, \ldots, x_{n}\right) \in \mathbf{R}^{n}$. Because the 'bipartite' wave function $\Psi$ of physical meaning should be 'Hermitian':

$$
\Psi^{*}(\vec{x}, \vec{y})=\Psi(\vec{y}, \vec{x})
$$

it is suitable to assume that

$$
F \in C(\bar{\Omega} \times \bar{\Omega}) \cap C^{2}(\Omega \times \Omega), \quad \overline{F(x, y)}=F(y, x),
$$

for all $x, y \in \bar{\Omega}$. In this case, letting

$$
W(x, y)=\left(\triangle_{x}-\triangle_{y}\right) F(x, y),
$$


for all $x, y \in \Omega$, we conclude that the Dirichlet problem (1.7) reduces to

$$
\left\{\begin{array}{l}
\left(-\triangle_{x}+\triangle_{y}\right) \Theta(x, y)=W(x, y), \quad x, y \in \Omega, \\
\left.\Theta\right|_{\partial \Omega \times \partial \Omega}=0,
\end{array}\right.
$$

where $\Theta(x, y)=\Phi(x, y)-F(x, y)$.

To solve (1.11) we will involve Hilbert-space's argument. Contrary to Laplace equation, the "energy functional" of (1.11) is not positive and so we shall utilize some additional techniques. To this end, anti-inner product spaces are defined and studied in Section 2, for which a Riesz-type representation theorem is proved. Finally, in Section 3 we prove the existence and uniqueness of the generalized solution of Dirichlet problem (1.11).

\section{Anti-inner product spaces}

In our terminology, an inner product on a vector space $\mathbf{V}$ over the field $\mathbf{C}$ (or, $\mathrm{R}$ ) is a complex-valued function $(\cdot, \cdot)$ defined for all pairs $x, y \in \mathbf{V}$ so that the conditions

$$
(\alpha x+\beta y, z)=\alpha(x, z)+\beta(y, z), \overline{(x, y)}=(y, x),
$$

are fulfilled for all $x, y, z \in \mathbf{V}$ and $\alpha, \beta \in \mathrm{C}$ (or, R). If $(x, x) \geq 0$ for all $x \in \mathbf{V}$ and $(x, x)=0$ only if $x=0$, the inner $(\cdot, \cdot)$ is said to be definite; otherwise indefinite. A vector space $\mathbf{V}$ over the field $\mathrm{C}$ (or, $\mathrm{R}$ ) equipped with a definite (or, indefinite) inner product is called a complex (or, real) inner (or, indefinite inner) product space (for details, see [1]). It is well known that a complex (or, real) inner product space $\mathbf{V}$ is a

complex (or, real) normed vector space under the norm $\|x\|=\sqrt{(x, x)}$, and said to be a complex (or, real) Hilbert space if it is complete in this norm.

Definition 2.1 An anti-inner product on a vector space $\mathbf{V}$ over the field $\mathrm{C}$ (or, $\mathrm{R}$ ) is a complex-valued function $\langle\cdot, \cdot\rangle$ defined for all pairs $x, y \in \mathbf{V}$ so that the conditions

$$
\langle\alpha x+\beta y, z\rangle=\alpha\langle x, z\rangle+\beta\langle y, z\rangle, \quad\langle x, y\rangle=-\langle y, x\rangle,
$$

are fulfilled for all $x, y, z \in \mathbf{V}$ and $\alpha, \beta \in \mathrm{C}$ (or, $\mathrm{R}$ ).

A vector space $\mathbf{V}$ over the field $\mathrm{C}$ (or, $\mathrm{R}$ ) equipped with an anti-inner product is called a complex (or, real) anti-inner product space.

An anti-inner product space $\mathbf{V}$ is called separated, if for every $x \in \mathbf{V} \backslash\{0\}$ there is a $y \in \mathbf{V}$ so that $\langle x, y\rangle \neq 0$.

Throughout the paper, when not specified, we assume that all anti-inner product spaces considered are separated.

Example 2.1 For $u, v \in \mathbf{C}^{2}$, define

$$
\langle u, v\rangle=\left|\begin{array}{ll}
a & b \\
c & d
\end{array}\right|
$$


where $u=(a, b), v=(c, d)$. Then, $\mathbf{C}^{2}$ with (2.2) is a complex anti-inner product space. Similarly, we can define an anti-inner product on $\mathbf{R}^{2}$ so that $\mathbf{R}^{2}$ becomes a real anti-inner product space.

Example 2.2 Let $\mathrm{V}$ be a complex inner (or, indefinite inner) product space with the inner product $(\cdot, \cdot)$. Define

$$
\langle x, y\rangle=(x, y)-(y, x)=2 i \operatorname{Im}(x, y)
$$

for all $x, y \in \mathbf{V}$. Then, $\mathbf{V}$ with $(2.3)$ is a real anti-inner product space. Here and afterwards, $\operatorname{Re}(a+i b)=a$ as well as $\operatorname{Im}(a+i b)=b$ for all $a, b \in \mathrm{R}$.

Note that, in this case, $\mathbf{V}$ with (2.3) cannot be a complex anti-inner product space. Indeed, by $(2.3)\langle x, y\rangle$ is purely imaginary or zero. Suppose that $\mathbf{V}$ were a complex anti-inner product space. Let $\langle x, y\rangle \neq 0$. We have

$$
\langle i x, i y\rangle=i\langle x, i y\rangle=-i\langle i y, x\rangle=-\langle x, y\rangle \neq 0 .
$$

On the other hand, $\langle x, i y\rangle$ is a purely imaginary number and so $i\langle x, i y\rangle$ is a real number. Hence, $\langle i x, i y\rangle=0$. This concludes a contradiction.

The anti-inner $(2.3)$ is determined by the inner product of $\mathbf{V}$, denoted by $\langle x, y\rangle_{\mathbf{V}}$ in the following.

Definition 2.2 An anti-inner product space $\mathbf{V}$ is said to be complete, if there is a complex Hilbert space $\mathbf{H}$ so that $\mathbf{V}$ is a real linear closed subspace of $\mathbf{H}$ with the anti-inner product $\langle\cdot, \cdot\rangle_{\mathbf{V}}=2 i \operatorname{Im}(\cdot, \cdot)_{\mathbf{H}}$.

A real linear functional on $\mathbf{V}$ is said to be bounded, if it is bounded in the norm determined by $(\cdot, \cdot)_{\mathbf{H}}$.

A linear functional $l$ on $\mathbf{V}$ is said to be representable, if there is a $y \in \mathbf{V}$ so that

$$
l(x)=\langle x, y\rangle_{\mathbf{V}}
$$

for all $x \in \mathbf{V}$.

Proposition 2.1 Let $\mathbf{V}$ be an anti-inner product space. Then

(i) $\langle z, \alpha x+\beta y\rangle=\alpha\langle z, x\rangle+\beta\langle z, y\rangle$ for all $x, y, z \in \mathbf{V}$ and $\alpha, \beta \in \mathrm{C}$ (or, R).

(ii) For every $x \in \mathbf{V},\langle x, x\rangle=0$.

(iii) Let $\mathbf{H}$ be a complex Hilbert space. Then, every real closed subspace of $\mathbf{H}$ is a complete anti-inner product space with the anti-inner product determined by $\langle\cdot, \cdot\rangle_{\mathbf{H}}$.

Proof. By definition, the results are immediate.

Proposition 2.2 Let $\mathrm{V}$ be a complete anti-inner product space determined by a complex Hilbert space $\mathbf{H}$. Then, a bounded real linear functional $l$ on $\mathbf{V}$ is representable if and only if $l$ is purely imaginary, that is, $\operatorname{Re} l(x)=0$ for all $x \in \mathbf{V}$. 
Stationary von Neumann-Landau wave equations

Proof. By Definition 2.2, the necessity is evident. To prove the sufficiency, we note that $\mathbf{V}$ is a closed subspace of $\mathbf{H}$ and so a real Hilbert space. Then, by Riesz's theorem there exists a $y \in \mathbf{V}$ such that

$$
l(x)=(x, y)_{\mathbf{H}}
$$

for all $x \in \mathbf{V}$. Since $l$ is purely imaginary, it is concluded that $l(x)=i \operatorname{Im}(x, y)$ for every $x \in \mathbf{V}$. This completes the proof.

\section{The main result}

As is well known, a variety of methods have been invented to solve the Dirichlet problem for the Laplace equation [5]. Among these is the method reducing the Dirichlet problem to a standard problem in Hilbert space, that make little use of special features of the Laplace equation, and can be extended to other problems and other equations. In the sequel, we will generalize the Hilbert-space method to solve the Dirichlet problem for the stationary von Neumann-Landau equation. Typically the solution of the Dirichlet problem proceeds in two steps by Hilbert-space method. In the first step a modified ("generalized") Dirichlet problem is solved in a deceptively simple manner. The second step consists in showing that under suitable regularity assumptions on region and data the "generalized" solution of the modified problem actually is a solution of the original problem. The second step, which involves more technical difficulties, will be carried out in another paper. From the point of view of applications one might even take the attitude that the modified problem already adequately describes the physical situation.

We now reformulate the Dirichlet problem (1.7) for the stationary von NeumannLandau equation as the problem of representing a certain bounded linear functional $l$ in a complete anti-inner product space as an anti-inner product $\langle\Psi, \Phi\rangle$. Under the condition (1.9), it suffices to consider (1.11) instead of (1.7).

In the space of functions of class $C^{1}(\bar{\Omega} \times \bar{\Omega})$ we define two bilinear forms $(\Psi, \Phi)$ and $\langle\Psi, \Phi\rangle$ by

$$
(\Psi, \Phi)=\int_{\Omega \times \Omega} \sum_{j=1}^{n} \frac{\partial \Psi}{\partial x_{j}} \frac{\overline{\partial \Phi}}{\partial x_{j}} d x d y+\int_{\Omega \times \Omega} \sum_{j=1}^{n} \frac{\partial \Psi}{\partial y_{j}} \frac{\overline{\partial \Phi}}{\partial y_{j}} d x d y
$$

and

$$
\langle\Psi, \Phi\rangle=\int_{\Omega \times \Omega} \sum_{j=1}^{n} \frac{\partial \Psi}{\partial x_{j}} \frac{\overline{\partial \Phi}}{\partial x_{j}} d x d y-\int_{\Omega \times \Omega} \sum_{j=1}^{n} \frac{\partial \Psi}{\partial y_{j}} \frac{\overline{\partial \Phi}}{\partial y_{j}} d x d y,
$$

respectively. Define $C_{0}^{1}(\bar{\Omega} \times \bar{\Omega})$ by

$$
C_{0}^{1}(\bar{\Omega} \times \bar{\Omega})=\left\{\Psi \in C^{1}(\bar{\Omega} \times \bar{\Omega}):\left.\Psi\right|_{\partial \Omega \times \partial \Omega}=0\right\} .
$$

Then, $C_{0}^{1}(\bar{\Omega} \times \bar{\Omega})$ with $(3.1)$ is a complex inner product space with the corresponding norm given by the Dirichlet integral

$$
\|\Psi\|=\sqrt{(\Psi, \Psi)}=\left(\int_{\Omega \times \Omega}\left[\sum_{j=1}^{n}\left|\frac{\partial \Psi}{\partial x_{j}}\right|^{2}+\sum_{j=1}^{n}\left|\frac{\partial \Psi}{\partial y_{j}}\right|^{2}\right] d x d y\right)^{1 / 2} .
$$


We complete $C_{0}^{1}(\bar{\Omega} \times \bar{\Omega})$ into a complex Hilbert space $H_{0}^{1}(\Omega \times \Omega)$ with respect to the Dirichlet norm (3.4).

On the other hand, define $S_{0}^{1}(\Omega \times \Omega)$ by

$$
S_{0}^{1}(\Omega \times \Omega)=\left\{\Psi \in H_{0}^{1}(\Omega \times \Omega): \overline{\Psi(x, y)}=\Psi(y, x) \text {, a.e. }(x, y) \in \Omega \times \Omega\right\} .
$$

Then, $S_{0}^{1}(\Omega \times \Omega)$ is a real closed subspace of $H_{0}^{1}(\Omega \times \Omega)$. For $\Psi, \Phi \in S_{0}^{1}(\Omega \times \Omega)$ define $(\Psi, \Phi)_{S}$ by

$$
(\Psi, \Phi)_{S}=\int_{\Omega \times \Omega} \sum_{j=1}^{n} \frac{\partial \Psi}{\partial x_{j}} \frac{\overline{\partial \Phi}}{\partial x_{j}} d x d y .
$$

By Fubini's theorem we have

$$
\begin{aligned}
& (\Psi, \Phi)_{S}=\int_{\Omega} \int_{\Omega} \sum_{j=1}^{n} \frac{\partial \Psi(x, y)}{\partial x_{j}} \frac{\overline{\partial \Phi(x, y)}}{\partial x_{j}} d x d y \\
& =\overline{\int_{\Omega} \int_{\Omega} \sum_{j=1}^{n} \frac{\partial \Psi(y, x)}{\partial x_{j}} \frac{\partial \overline{\Phi(y, x)}}{\partial x_{j}} d x d y} \\
& =\overline{\int_{\Omega} \int_{\Omega} \sum_{j=1}^{n} \frac{\partial \Psi(y, x)}{\partial x_{j}} \frac{\partial \overline{\Phi(y, x)}}{\partial x_{j}} d y d x} \\
& =\overline{\int_{\Omega} \int_{\Omega} \sum_{j=1}^{n} \frac{\partial \Psi(x, y)}{\partial y_{j}} \frac{\overline{\partial \Phi(x, y)}}{\partial y_{j}} d x d y} .
\end{aligned}
$$

Hence, for $\Psi, \Phi \in S_{0}^{1}(\Omega \times \Omega)$ we have

$$
(\Psi, \Phi)=(\Psi, \Phi)_{S}+(\Phi, \Psi)_{S}
$$

and

$$
\langle\Psi, \Phi\rangle=(\Psi, \Phi)_{S}-(\Phi, \Psi)_{S}
$$

In particular,

$$
\|\Psi\|=\sqrt{2}\|\Psi\|_{S}
$$

where $\|\Psi\|_{S}=\sqrt{(\Psi, \Psi)_{S}}$. This means that $S_{0}^{1}(\Omega \times \Omega)$ with (3.2) is a real anti-inner product space. Since $S_{0}^{1}(\Omega \times \Omega)$ is a real closed subspace of $H_{0}^{1}(\Omega \times \Omega)$, by the Projection theorem we have that

$$
H_{0}^{1}(\Omega \times \Omega)=S_{0}^{1}(\Omega \times \Omega) \oplus S_{0}^{1}(\Omega \times \Omega)^{\perp}
$$

Set $\mathbf{H}=H_{0}^{1}(\Omega \times \Omega)$ with the inner product $(\cdot, \cdot)_{\mathbf{H}}$ defined by

$$
(\Psi, \Phi)_{\mathbf{H}}=\left(\Psi_{1}, \Phi_{1}\right)_{S}+\left(\Psi_{2}, \Phi_{2}\right)
$$

for $\Psi=\Psi_{1}+\Psi_{2}, \Phi=\Phi_{1}+\Phi_{2} \in \mathbf{H}$ with

$$
\Psi_{1}, \Phi_{1} \in S_{0}^{1}(\Omega \times \Omega), \quad \Psi_{2}, \Phi_{2} \in S_{0}^{1}(\Omega \times \Omega)^{\perp} .
$$

By (3.9) we have

Proposition 3.1 Set $\mathbf{V}=S_{0}^{1}(\Omega \times \Omega)$. Then, $\mathbf{V}$ with (3.2) is a complete anti-inner product space determined by the Hilbert space $\mathbf{H}$ with the inner product (3.10). 
Stationary von Neumann-Landau wave equations

Let $\Theta \in C^{2}(\bar{\Omega} \times \bar{\Omega})$ be a solution of (1.11), where the prescribed $W$ belongs to $C(\bar{\Omega} \times \bar{\Omega})$. Then, for any $\Psi \in C_{0}^{1}(\bar{\Omega} \times \bar{\Omega})$ we have by the divergence theorem

$$
\begin{aligned}
\langle\Psi, \Theta\rangle & =\int_{\Omega \times \Omega} \Psi(x, y)\left(-\Delta_{x}+\Delta_{y}\right) \overline{\Theta(x, y)} d x d y \\
& =\int_{\Omega \times \Omega} \Psi(x, y) \overline{W(x, y)} d x d y .
\end{aligned}
$$

This suggests that $\Theta$ can be found by simply representing the known linear functional

$$
l(\Psi)=\int_{\Omega \times \Omega} \Psi(x, y) \overline{W(x, y)} d x d y
$$

as an anti-inner product $\langle\Psi, \Theta\rangle$. Our modified version (generalized solution) of the Dirichlet problem (1.11) is then the following:

Definition 3.1 If $\Theta \in S_{0}^{1}(\Omega \times \Omega)$ such that

$$
\langle\Psi, \Theta\rangle=l(\Psi)
$$

for all $\Psi \in S_{0}^{1}(\Omega \times \Omega)$, where $\langle\Psi, \Theta\rangle$ and $l(\Psi)$ are defined by (3.2) and (3.12) respectively, then $\Theta$ is said to be the generalized solution of the Dirichlet problem (1.11).

As following is the main result of the paper.

Theorem 3.1 Suppose $W \in L^{2}(\Omega \times \Omega)$ so that $\overline{W(x, y)}=-W(y, x)$ for almost all $(x, y) \in \Omega \times \Omega$. Then, there exists a unique generalized solution for the Dirichlet problem (1.11).

Proof. First, we have to show that the functional $l$ is bounded. Since by the Cauchy-Schwartz inequality

$$
|l(\Psi)|^{2}=\left|\int_{\Omega \times \Omega} \Psi(x, y) \overline{W(x, y)} d x d y\right|^{2} \leq \int_{\Omega \times \Omega}|\Psi(x, y)|^{2} d x d y \int_{\Omega \times \Omega}|W(x, y)|^{2} d x d y,
$$

and by Poincaré's inequality that there is a constant $M>0$ such that

$$
\int_{\Omega \times \Omega}|\Psi(x, y)|^{2} d x d y \leq M \int_{\Omega \times \Omega}\left[\sum_{j=1}^{n}\left|\frac{\partial \Psi}{\partial x_{j}}\right|^{2}+\sum_{j=1}^{n}\left|\frac{\partial \Psi}{\partial y_{j}}\right|^{2}\right] d x d y,
$$

for all $\Psi \in H_{0}^{1}(\Omega \times \Omega)$, we conclude by (3.9) that $l$ is a bounded linear functional on $S_{0}^{1}(\Omega \times \Omega)$.

Since $\overline{W(x, y)}=-W(y, x)$ for almost all $(x, y) \in \Omega \times \Omega$, we have by Fubini's theorem

$$
\begin{aligned}
\overline{l(\Psi)} & =\int_{\Omega \times \Omega} \overline{\Psi(x, y)} W(x, y) d x d y \\
& =-\int_{\Omega \times \Omega} \Psi(y, x) \overline{W(y, x)} d x d y \\
& =-\int_{\Omega \times \Omega} \Psi(y, x) \overline{W(y, x)} d y d x \\
& =-l(\Psi)
\end{aligned}
$$


for every $\Psi \in S_{0}^{1}(\Omega \times \Omega)$. This concludes that $l$ is purely imaginary. Therefore, by Proposition 2.2 we prove the existence of the generalized solution. The uniqueness of the generalized solution follows immediately from the Riesz representation theorem. The proof is complete.

For $f \in C^{2}(\bar{\Omega})$, set $F(x, y)=f(x) f(y)$. Then, $\Phi(x, y)=u(x) u(y)$ satisfies $(1.7)$, where $u$ is the solution of the Dirichlet problem for Laplace equation:

$$
\left\{\begin{array}{l}
-\triangle u(x)=0, \quad x \in \Omega, \\
\left.u\right|_{\partial \Omega}=f .
\end{array}\right.
$$

Generally speaking, $F$ cannot be written as the product form of $F(x, y)=f(x) f(y)$, hence our results extend the classical results for Laplace equation.

\section{Acknowledgments}

This work was partially supported by the National Natural Science Foundation of China under Grant No.10571176.

\section{References}

[1] Bognár J 1974 Indefinite inner product spaces (Springer-Verlag, Berlin Heidelberg New York)

[2] Chen Zeqian 2007 von Neumann-Landau equation for wave functions, wave-particle duality and collapses of wave functions. quant-ph/0703204

[3] Dirac P A M 1958 The Principles of Quantum Mechanics (Fourth edition, Oxford University Press, Oxford)

[4] Fan H Y and Li C 2004 Phys.Lett.A 32175

[5] John F 1982 Partial differential equations (Fourth edition, Springer-Verlag, New York)

[6] Landau L D and Lifshitz E M 1977 Quantum Mechanics (Non-relativistic Theory) (Third edition, Pergamon Press, Oxford)

[7] von Neumann J 1955 Mathematical Foundations of Quantum Mechanics (Princeton University Press, Princeton) 